needlestick injuries that has not been assessed in a trial.' Should not their dogmatic approach follow, rather than precede, publication of data that support non-recapping? There is no published evidence to show that non-recapping reduces the incidence of needlestick injury. As Professor D C Anderson and colleagues rightly point out, the Needle Guard significantly reduces the rate of needlestick injury associated with venepuncture. ${ }^{2}$

It is a matter of concern that people working in a clinical trials centre choose to ignore published data and fail to present new data to corroborate their views.

PAUL N GOLDWATER

Microbiology Department

Adelaide Children's Hospital,

North Adelaide,

South Australia 5006

1 Valentine C, Bright P. Preventing needlestick injuries. $B M \mathcal{f}$ 1991;302:1079. (4 May.)

2 Anderson DC, Blower AL, Packer JMV, Ganguli LA. Preventing needlestick injuries. BMF 1991;302:769-70. (30 March.)

\section{Tuberculosis and HIV infection}

SIR, - We were surprised by the results quoted in Dr David Inwald's letter, ' which suggested that the rate of positivity for HIV-I among patients with pulmonary tuberculosis in southern Africa was significantly lower than the $40 \cdot 2 \%$ positivity rate for HIV-I and HIV-II found by Dr Kevin M De Cock in the Ivory Coast. ${ }^{2}$

On 1 February this year we began a two year prospective study of the rate of positivity for HIV-I and HIV-II among all new patients with pulmonary tuberculosis presenting to a regional hospital in eastern Swaziland. In contrast to Dr Inwald's findings of a positivity rate of $7 \cdot 2 \%$ (five out of 69 subjects), our results so far suggest a rate of $29 \cdot 1 \%$ ( 21 out of 72 subjects; results obtained with enzyme linked immunosorbent assay (ELISA) and confirmed by virus specific western blotting). This unfortunately suggests that it is now too late "to nip the epidemic in the bud."

Dr Inwald dismisses measures to prevent AIDS as being non-existent, but there is an extensive prevention campaign coordinated by the government of Swaziland with funding from and the close support of the World Health Organisation, the European Community, and USAID. One interesting aspect of this is the successful recruitment of craditional healers ("witchdoctors") in increasing awareness of AIDS among the rural population.

JOHN WRIGHT HELEN FORD

Good Shepherd Hospital

PO Box 2, Siteki

Swaziland

1 Inwald D. Tuberculosis and HIV infection. BMf 1991;302:726. (23 March.)

2 De Cock KM, Gnaore E, Adjorlolo G, Braun MM, Lafontaine $M-F$, Yesso G, et al. Risk of tuberculosis in patients with HIV-I and HIV-II infections in Abidjan, Ivory Coast. BMJ 1991;302: 496-9. (2 March.)

\section{Spontaneous pneumothorax}

SIR,-Dr Douglas Seaton and his colleagues may have made an important contribution to the management of spontaneous pneumothorax, 'but I agree with Dr I Lenox-Smith that their results should be compared with those obtained with the machines he mentions. ${ }^{2}$

The Maxwell box can still be bought - at a cost of $£ 620$ +VAT - from Thackray, but some years ago I made a manometer at little expense by mounting a glass $U$ tube containing coloured water on a board marked in centimetres. Junior doctors were trained to recognise the characteristic pressures found in closed, open, and tension pneumothoraces and to decide whether to aspirate air or to intubate. Using a syringe and a two way tap to aspirate air gave sastisfactory results. The Stott or Maxwell apparatus enables the procedure to be completed more quickly and easily and ought to be available in medical wards.

J B MORRISON

Southport,

Merseyside PR8 6PJ

1 Seaton D, Yoganathan K, Coady T, Barker R. Spontaneous pneumothorax: marker gas technique for predicting outcom of manual aspiration. BMf 1991;302:262-5. (2 February.) 2 Lenox-Smith I. Spontaneous pneumothorax. BMF 1991;302:595. (9March.)

\section{The cyclotron saga continues}

SIR,-Using fast neutrons to treat large hypoxic tumours makes good radiobiological sense. Unfortunately, the clinical data on their merits relative to those of conventional and cheaper radiotherapy are confusing.

Despite its dramatic title the recent paper from the group using Britain's only cyclotron available for treating cancer does not help. ${ }^{1}$ Amid the elegant statistical analysis is hidden the remarkable observation that the group of patients treated with neutrons had a high rate of relapse with metastatic disease within a year of treatment. The pelvic tumours studied were predominantly slow growing. This implies the presence of metastases at the time of treatment. It is therefore not a fair test of the efficacy of neutrons. We cannot, as many would like, dismiss neutrons so easily.

Huge sums of money have been spent on the Clatterbridge project by the Medical Research Council, cancer charities, and the NHS. The tota amount almost certainly exceeds $£ 12 \mathrm{~m}$ and may be as much as $£ 20 \mathrm{~m}$. For the sake of future patients we need to know the truth about neutrons. This paper does not tell us the whole story. It is perhaps significant that the two successive senior clinical coordinators of the trial in Liverpool are not included among the paper's authors.

Department of Clinical Oncology,

KAROL SIKORA

Department of Clinical Oncology,

Rayal Postgraduate Medical

London W12 0HS

1 Errington RD, Ashby D, Gore SM, Abrams KR, Myint S, Bonnett $\mathrm{D}$, et al. High energy neutron treatment for pelvic cancers: study stopped because of increased mortality. $B M Y$ 1991;302:1045-51. (4 May.)

AUTHOR'S REPLY, - Using neutrons to treat large hypoxic tumours might make good radiobiologica sense, but this cannot be extrapolated directly to imply good clinical sense when radiobiologists recognise the problem of metastatic disease even if improved local control is achieved. ${ }^{\prime}$ When locally advanced tumours are being treated the problem of excess damage to normal tissue must also be considered, whether this results from direct effects of the tumour or the effects of radiation treatment.

If Professor Sikora has any substantial personal experience of treating patients with locally advanced cancer in properly designed randomised trials I am surprised that he does not more readily acknowledge the problems encountered with such patients due to relapse with metastatic disease. Even when full tumour staging procedures are carried out before randomisation it is implicit tha occult metastatic disease will present in some patients entered into studies such as the trial of neutrons in pelvic cancer. ${ }^{2}$ There was, however, no bias towards entering these patients into the neutron or photon group. It is totally unrealistic to dismiss the trial as not being a fair test of the efficacy of neutrons.

It is not clear how Professor Sikora wishes to relate his unsubstantiated statement about the pelvic tumours studied being predominantly slow growing to their metastatic potential. Is he really saying that all the tumours in this study should have a low metastatic potential and a low risk of occult metastatic disease being present at the time of definitive radical local treatment? If this is the case perhaps he could explain why in current randomised trials of locally advanced bladder cancer the role of neoadjuvant chemotherapy is being tested. ${ }^{3}$ The problem of occult metastatic disease is also recognised in rectal cancer, and adjuvant local and systemic treatment is now advocated. ${ }^{4}$ It is unrealistic to expect complete results from a randomised trial to be published within two years of the recruitment of patients being stopped. It was important to explain why the study of neutron treatment in pelvic cancer was stopped, and the paper does this. Neutrons have not been dismissed easily in any role they may have been thought to have in advanced rectal, bladder, or cervical cancer. When further follow up data have accrued a final report will be published.

I agree that we need to know the truth about neutrons, and good clinical research practice demands that this truth should be sought from randomised clinical trials. In view of the contributions from Professor Sikora's colleagues to the debate on neutrons ${ }^{56} \mathrm{I}$ am surprised that he has not been more involved in documenting the whole story of Hammersmith's experience of neutron treatment, where surely input from a radiation oncologist is necessary in completing the descriptions of the late morbidity in patients treated with neutrons.

In answer to his final point, the current clinical coordinator acknowledges that the authors of the paper comprised colleagues who made the most significant clinical and technical contributions to the trial. The paper and its authorship in the form published were approved by the cancer therapy committee of the Medical Research Council.

Mersey Regional Centre for Radiotherapy

R D ERRINGTON

$$
\text { and Oncology, }
$$

Clatterbridge Hospital

Bebington,

Wirral,

Merseyside L634JY

1 Field SB. An historical survey of radiobiology and radiotherapy with fast neutrons. Current Topics in Radiation Research Quarterly 1976;11:1-86.

2 Errington RD, Ashby D, Gore SM, Abrams KR, Myint S, Bonnett $\mathrm{DE}$, et al. High energy neutron treatment for pelvic cancers: study stopped because of increased mortality. BMJ cancers: study stopped because of increased mortality. $B M$ 1991,302:1045-51. (4 May.)

3 Medical Research Council and European Organisation for the Research and Treatment of Cancer. A randomised trial of primary chemotherapy in locally advanced bladder cancer. London: MRC, October 1989. (MRC protocol BA06; EORTC protocol 30894 .

4 Krook JE, Moertel CG, Gunderson LL, Wieand HS, Collins RT, Beart RW, et al. Effective surgical adjuvant therapy for highrisk rectal carcinoma. $N$ Engl f Med 1991;324:709-15.

Davies DM, Stafford ND, Smith R. Radiotherapy's second setback. BMF 1989:298:183.

6 Stafford N, Waldron J, Davies D, Smith R. Fast neutron treatment for squamous cell carcinoma. $B M \mathcal{J}$ 1991;302:48. (5 January.)

\section{Use of thalidomide in leprosy}

SIR, - In discussing the management of erythema nodosum leprosum Dr Adam S Malin and colleagues describe the side effects of corticosteroids and clofazimine. ${ }^{1}$ As they do not mention that thalidomide, the third drug discussed, has any side effects it might be assumed that thalidomide is safe to use provided, of course, that it is not given to women of reproductive age. This, however, is not the case as the drug causes "peripheral neuropathies which can be severe and irreversible."”

The implication that low maintenance doses (50-100 $\mathrm{mg}$ daily $)^{3}$ may be safe is equally misleading. Patients who took the drug in this dosage as a sedative developed a neuropathy, and loss of nerve fibre was confirmed histologically even 